\title{
A FAST SPECTRAL ALGORITHM FOR THE QUANTUM BOLTZMANN COLLISION OPERATOR*
}

\author{
JINGWEI HU ${ }^{\dagger}$ AND LEXING YING ${ }^{\ddagger}$
}

\begin{abstract}
This paper introduces a fast spectral algorithm for the quantum Boltzmann collision operator. In the usual spectral framework, one of the terms in the operator cannot be evaluated efficiently. The new approach is based on the fundamental property of the exponential function which allows one to construct a new decomposition of the collision kernel to speed up the computation. Numerical results in 2-D and 3-D for both the Bose gas and the Fermi gas are presented to illustrate the accuracy and efficiency of the method.
\end{abstract}

Key words. Quantum Boltzmann equation, fast spectral method.

AMS subject classifications. 35Q20, 65M70.

\section{Introduction}

The quantum Boltzmann equation describes the time evolution of a dilute Bose gas or Fermi gas. It was first formulated by Nordheim [13] and Uehling and Uhlenbeck [17] from the classical Boltzmann equation by heuristic arguments. If $f(t, x, v)$ is the phase space distribution function of time $t$, position $x$, and particle velocity $v$, then the equation reads

$$
\frac{\partial f}{\partial t}+v \cdot \nabla_{x} f=\mathcal{Q}_{q}(f), \quad x \in \Omega \subset \mathbb{R}^{d_{x}}, v \in \mathbb{R}^{d_{v}} .
$$

The quantum collision operator $\mathcal{Q}_{q}$ is given by

$$
\begin{array}{r}
\mathcal{Q}_{q}(f)(v)=\int_{\mathbb{R}^{d v}} \int_{\mathbb{S}^{d_{v}-1}} B\left(v-v_{*}, \omega\right)\left[f^{\prime} f_{*}^{\prime}\left(1 \pm \theta_{0} f\right)\left(1 \pm \theta_{0} f_{*}\right)\right. \\
\left.-f f_{*}\left(1 \pm \theta_{0} f^{\prime}\right)\left(1 \pm \theta_{0} f_{*}^{\prime}\right)\right] d \omega d v_{*},
\end{array}
$$

where $\theta_{0}=h^{d_{v}}$ and $h$ is the rescaled Planck constant. In this paper, the upper sign will always correspond to the Bose gas while the lower sign to the Fermi gas. $f, f_{*}, f^{\prime}$, and $f_{*}^{\prime}$ are the shorthand notations for $f(t, x, v), f\left(t, x, v_{*}\right), f\left(t, x, v^{\prime}\right)$, and $f\left(t, x, v_{*}^{\prime}\right)$ respectively. $\left(v, v_{*}\right)$ and $\left(v^{\prime}, v_{*}^{\prime}\right)$ are the velocities before and after collision. They are related by the following parametrization:

$$
\left\{\begin{array}{l}
v^{\prime}=\frac{v+v_{*}}{2}+\frac{\left|v-v_{*}\right|}{2} \omega \\
v_{*}^{\prime}=\frac{v+v_{*}}{2}-\frac{\left|v-v_{*}\right|}{2} \omega
\end{array}\right.
$$

${ }^{*}$ Received: September 7, 2011; accepted (in revised version): October 14, 2011. Communicated by Shi Jin.

J.H. would like to thank KAUST for their generous support. L.Y. was supported in part by the NSF under CAREER grant DMS-0846501.

${ }^{\dagger}$ Institute for Computational Engineering and Sciences (ICES), The University of Texas at Austin, 1 University Station C0200, Austin, TX 78712, USA (hu@ices.utexas.edu).

${ }^{\ddagger}$ Department of Mathematics and ICES, The University of Texas at Austin, 1 University Station C1200, Austin, TX 78712, USA (lexing@math.utexas.edu). 
where $\omega$ is the unit vector along $v^{\prime}-v_{*}^{\prime}$. The collision kernel $B$ is a nonnegative function that only depends on $\left|v-v_{*}\right|$ and $\cos \theta=w \cdot\left(v-v_{*}\right) /\left|v-v_{*}\right|$. From now on we will write it as $B\left(\left|v-v_{*}\right|, \cos \theta\right)$.

The quantum collision operator $\mathcal{Q}_{q}$ satisfies the conservation of mass, momentum, and energy,

$$
\int_{\mathbb{R}^{d} v} \mathcal{Q}_{q}(f) d v=\int_{\mathbb{R}^{d_{v}}} \mathcal{Q}_{q}(f) v d v=\int_{\mathbb{R}^{d v}} \mathcal{Q}_{q}(f)|v|^{2} d v=0
$$

and Boltzmann's H-theorem

$$
\int_{\mathbb{R}^{d_{v}}} \ln \left(\frac{f}{1 \pm \theta_{0} f}\right) \mathcal{Q}_{q}(f) d v \leq 0 .
$$

Moreover, the equality in (1.5) holds if and only if $f$ attains the local equilibrium (the quantum Maxwellian):

$$
\mathcal{M}_{q}=\frac{1}{\theta_{0}} \frac{1}{z^{-1} e^{\frac{(v-u)^{2}}{2 T}} \mp 1},
$$

where $z$ is the fugacity, $T$ is the temperature, and $u$ is the macroscopic velocity. (1.6) is the well-known Bose-Einstein ('-') and Fermi-Dirac ('+') distributions. For further details about the derivation of $\mathcal{M}_{q}$ and the properties of the quantum Boltzmann equation we refer to $[9,5]$.

The numerical challenge of solving the Boltzmann equation mainly comes from the multidimensional structure of the collision integral. For the classical Boltzmann operator, many different approaches are available: for example, the direct simulation Monte Carlo (DSMC) methods [1, 12], the discrete velocity models (DVM) [16, 2, 4], and more recently the spectral methods $[3,14,15,11,6,7]$. On the contrary, very few studies have been conducted in the quantum case. See [8] and references therein for the Monte Carlo simulations and [10] for a fast deterministic scheme for the energy space boson Boltzmann equation.

In [5] the authors constructed a spectral method for the quantum Boltzmann collision operator (1.2) following the framework of $[11,6]$. However, due to its cubic structure, one of the terms in $\mathcal{Q}_{q}$ cannot be evaluated by the fast Fourier transform (FFT), which results in a computational cost of $O\left(M^{d_{v}-1} N^{2 d_{v}} \log N\right)(M$ is the number of points in angular direction; $N$ is the number of points in each $v$ direction). In this work, we improve the above method and develop a fast algorithm for the quantum Boltzmann operator based on a simple observation of the exponential function. The numerical cost is reduced to $O\left(M^{d_{v}-1} N^{d_{v}+1} \log N\right)$.

The rest of the paper is organized as follows: In the next section we first describe the spectral method in [5] and then present the new fast algorithm. Numerical results in both 2-D and 3-D are shown in Section 3 to illustrate the accuracy and efficiency of the method. Finally the concluding remarks are given in Section 4.

2. The spectral methods for the quantum Boltzmann collision operator

We begin by a brief description of the spectral method in [5]. It serves as the starting point of our fast algorithm and as a comparison in the numerical tests. For simplicity, we drop the spatial dependence and consider the homogeneous quantum Boltzmann equation:

$$
\frac{\partial f}{\partial t}=\mathcal{Q}_{q}(f), \quad v \in \mathbb{R}^{d_{v}} .
$$


2.1. First attempt. The quantum collision operator $\mathcal{Q}_{q}$ can be recast as

$$
\mathcal{Q}_{q}=\mathcal{Q}_{c} \pm \theta_{0}\left(\mathcal{Q}_{1}+\mathcal{Q}_{2}-\mathcal{Q}_{3}-\mathcal{Q}_{4}\right)
$$

where

$$
\mathcal{Q}_{c}(f)(v)=\int_{\mathbb{R}^{d v}} \int_{\mathbb{S}^{d v}-1} B\left(\left|v-v_{*}\right|, \cos \theta\right)\left[f^{\prime} f_{*}^{\prime}-f f_{*}\right] d \omega d v_{*}
$$

is the classical collision operator. The cubic terms $\mathcal{Q}_{1}-\mathcal{Q}_{4}$ are

$$
\left\{\begin{array}{l}
\mathcal{Q}_{1}(f)(v)=\int_{\mathbb{R}^{d v}} \int_{\mathbb{S}^{d^{2}-1}} B\left(\left|v-v_{*}\right|, \cos \theta\right) f^{\prime} f_{*}^{\prime} f_{*} d \omega d v_{*} \\
\mathcal{Q}_{2}(f)(v)=\int_{\mathbb{R}^{d_{v}}} \int_{\mathbb{S}^{d_{v}-1}} B\left(\left|v-v_{*}\right|, \cos \theta\right) f^{\prime} f_{*}^{\prime} f d \omega d v_{*} \\
\mathcal{Q}_{3}(f)(v)=\int_{\mathbb{R}^{d_{v}}} \int_{\mathbb{S}^{d_{v}-1}} B\left(\left|v-v_{*}\right|, \cos \theta\right) f f_{*} f^{\prime} d \omega d v_{*} \\
\mathcal{Q}_{4}(f)(v)=\int_{\mathbb{R}^{d_{v}}} \int_{\mathbb{S}^{d^{2}-1}} B\left(\left|v-v_{*}\right|, \cos \theta\right) f f_{*} f_{*}^{\prime} d \omega d v_{*}
\end{array}\right.
$$

In order to perform the Fourier transform, we periodize the function $f$ on the domain $\mathcal{D}_{L}=[-L, L]^{d_{v}}$ ( $L$ is chosen such that $L \geq \frac{3+\sqrt{2}}{2} S$, where $\mathcal{B}_{S}$ is an approximation of the support of $f(v)[15])$. Using a Carleman like representation [11], one can transform the above operators to

$$
\mathcal{Q}_{c}(f)(v)=\int_{\mathcal{B}_{R}} \int_{\mathcal{B}_{R}} \tilde{B}(x, y) \delta(x \cdot y)[f(v+x) f(v+y)-f(v+x+y) f(v)] d x d y
$$

and

$$
\left\{\begin{array}{l}
\mathcal{Q}_{1}(f)(v)=\int_{\mathcal{B}_{R}} \int_{\mathcal{B}_{R}} \tilde{B}(x, y) \delta(x \cdot y) f(v+x) f(v+y) f(v+x+y) d x d y \\
\mathcal{Q}_{2}(f)(v)=\int_{\mathcal{B}_{R}} \int_{\mathcal{B}_{R}} \tilde{B}(x, y) \delta(x \cdot y) f(v+x) f(v+y) f(v) d x d y \\
\mathcal{Q}_{3}(f)(v)=\int_{\mathcal{B}_{R}} \int_{\mathcal{B}_{R}} \tilde{B}(x, y) \delta(x \cdot y) f(v+x) f(v+x+y) f(v) d x d y \\
\mathcal{Q}_{4}(f)(v)=\int_{\mathcal{B}_{R}} \int_{\mathcal{B}_{R}} \tilde{B}(x, y) \delta(x \cdot y) f(v+y) f(v+x+y) f(v) d x d y
\end{array}\right.
$$

where $\mathcal{B}_{R}$ is the truncation of the collision integral which satisfies $R \geq \sqrt{2} S$;

$$
\tilde{B}(x, y)=\frac{2^{d_{v}-1}}{\left(|x|^{2}+|y|^{2}\right)^{\frac{d_{v}-2}{2}}} B\left(\sqrt{|x|^{2}+|y|^{2}}, 1-\frac{2|x|^{2}}{|x|^{2}+|y|^{2}}\right)=\tilde{B}(|x|,|y|) .
$$

We approximate $f$ by a truncated Fourier series ( $k$ is the $d_{v}$-dimensional index):

$$
f(v) \approx \sum_{k=-\frac{N}{2}}^{\frac{N}{2}-1} \hat{f}_{k} e^{i \frac{\pi}{L} k \cdot v}, \quad \hat{f}_{k}=\frac{1}{(2 L)^{d_{v}}} \int_{\mathcal{D}_{L}} f(v) e^{-i \frac{\pi}{L} k \cdot v} d v
$$


Plugging it into $(2.1)$, one can get the following equation for $\hat{f}_{k}$ by the orthogonality of the basis $e^{i \frac{\pi}{L} k \cdot v}, k \in \mathbb{Z}^{d_{v}}$ :

$$
\frac{d \hat{f}_{k}}{d t}=\hat{\mathcal{Q}}_{q, k}=\hat{\mathcal{Q}}_{c, k} \pm \theta_{0}\left(\hat{\mathcal{Q}}_{1, k}+\hat{\mathcal{Q}}_{2, k}-\hat{\mathcal{Q}}_{3, k}-\hat{\mathcal{Q}}_{4, k}\right)
$$

where

$$
\hat{\mathcal{Q}}_{c, k}=\sum_{\substack{l, m=-\frac{N}{2} \\ l+m=k}}^{\frac{N}{2}-1}[\beta(l, m)-\beta(m, m)] \hat{f}_{l} \hat{f}_{m}
$$

and

$$
\left\{\begin{array}{c}
\hat{\mathcal{Q}}_{1, k}=\sum_{\substack{l, m, n=-\frac{N}{2} \\
l+m+n=k}}^{\frac{N}{2}-1} \beta(l+n, m+n) \hat{f}_{l} \hat{f}_{m} \hat{f}_{n}, \quad \hat{\mathcal{Q}}_{2, k}=\sum_{\substack{l, m, n=-\frac{N}{2} \\
l+m+n=k}}^{\frac{N}{2}-1} \beta(l, m) \hat{f}_{l} \hat{f}_{m} \hat{f}_{n}, \\
\hat{\mathcal{Q}}_{3, k}=\sum_{\substack{l, m, n=-\frac{N}{2} \\
l+m+n=k}}^{\frac{N}{2}-1} \beta(l+m, m) \hat{f}_{l} \hat{f}_{m} \hat{f}_{n}, \quad \hat{\mathcal{Q}}_{4, k}=\sum_{\substack{l, m, n=-\frac{N}{2} \\
l+m+n=k}}^{\frac{N}{2}-1} \beta(m, l+m) \hat{f}_{l} \hat{f}_{m} \hat{f}_{n},
\end{array}\right.
$$

with the kernel mode $\beta$ defined by

$$
\beta(l, m)=\int_{\mathcal{B}_{R}} \int_{\mathcal{B}_{R}} \tilde{B}(|x|,|y|) \delta(x \cdot y) e^{i \frac{\pi}{L} l \cdot x} e^{i \frac{\pi}{L} m \cdot y} d x d y .
$$

The classical part was treated in [11] by introducing a decomposition for $\beta$ :

$$
\beta(l, m) \approx \sum_{p=1}^{M^{d_{v}-1}} \alpha_{p}(l) \alpha_{p}^{\prime}(m)
$$

where $\alpha_{p}, \alpha_{p}^{\prime}$ are some functions to be given below.

Now with the same decomposition the remaining four cubic quantum terms can be approximated as

$$
\hat{\mathcal{Q}}_{1, k} \approx \sum_{p=1}^{M^{d_{v}-1}} \sum_{n=-\frac{N}{2}}^{\frac{N}{2}-1}\left[\sum_{\substack{l, m=-\frac{N}{2} \\ l+m=k-n}}^{\frac{N}{2}-1} \alpha_{p}(l+n) \alpha_{p}^{\prime}(m+n) \hat{f}_{l} \hat{f}_{m}\right] \hat{f}_{n}=\sum_{p=1}^{M^{d_{v}-1}} \sum_{n=-\frac{N}{2}}^{\frac{N}{2}-1} \hat{g}_{k-n}(n) \hat{f}_{n},
$$




$$
\begin{aligned}
& \hat{\mathcal{Q}}_{2, k} \approx \sum_{p=1}^{M^{d_{v}-1}} \sum_{n=-\frac{N}{2}}^{\frac{N}{2}-1}\left[\sum_{\substack{l, m=-\frac{N}{2} \\
l+m=k-n}}^{\frac{N}{2}-1} \alpha_{p}(l) \alpha_{p}^{\prime}(m) \hat{f}_{l} \hat{f}_{m}\right] \hat{f}_{n}, \\
& \hat{\mathcal{Q}}_{3, k} \approx \sum_{p=1}^{M^{d_{v}-1}} \sum_{n=-\frac{N}{2}}^{\frac{N}{2}-1} \alpha_{p}(k-n)\left[\sum_{\substack{l, m=-\frac{N}{2} \\
l+m=k-n}}^{\frac{N}{2}-1} \alpha_{p}^{\prime}(m) \hat{f}_{l} \hat{f}_{m}\right] \hat{f}_{n}, \\
& \hat{\mathcal{Q}}_{4, k} \approx \sum_{p=1}^{M^{d_{v}-1}} \sum_{n=-\frac{N}{2}}^{\frac{N}{2}-1} \alpha_{p}^{\prime}(k-n)\left[\sum_{\substack{l, m=-\frac{N}{2} \\
l+m=k}}^{\frac{N}{2}-1} \alpha_{p}(m) \hat{f}_{l} \hat{f}_{m}\right] \hat{f}_{n} .
\end{aligned}
$$

Thus, with the above arrangements, $\mathcal{Q}_{2}, \mathcal{Q}_{3}$, and $\mathcal{Q}_{4}$ (all in double-convolution forms) can be evaluated by the FFT in $O\left(M^{d_{v}-1} N^{d_{v}} \log N\right)$ operations. For instance, to compute $\mathcal{Q}_{3}$, we first treat $\hat{f}_{l}$ and $\alpha_{p}^{\prime}(m) \hat{f}_{m}$ as two functions, then terms inside the bracket of (2.16) form a convolution and can be evaluated as multiplication in the original space via the FFT. The resulting function (with subindex $k-n$ ) multiplied by $\alpha_{p}(k-n)$ together with $\hat{f}_{n}$ forms a convolution again and can be computed by the FFT as well. However, the fast algorithm cannot be applied to $\mathcal{Q}_{1}$, since $\hat{g}_{k-n}(n)$ itself depends on $n$ (although the terms inside the bracket are a convolution). We compute $\mathcal{Q}_{1}$ directly at the moment and the total computational cost is dominated by this part, which is $O\left(M^{d_{v}-1} N^{2 d_{v}} \log N\right)$.

2.1.1. The decomposition of $\beta$. We now discuss the decomposition (2.13) of $\beta$. Following [11], (2.12) becomes

$$
\begin{aligned}
\beta(l, m)=\frac{1}{4} & \int_{e, e^{\prime} \in \mathbb{S}^{d_{v}-1}} \delta\left(e \cdot e^{\prime}\right) d e d e^{\prime} \\
& \cdot \int_{-R}^{R} \int_{-R}^{R} \tilde{B}\left(|\rho|,\left|\rho^{\prime}\right|\right) e^{i \frac{\pi}{L} \rho(l \cdot e)} e^{i \frac{\pi}{L} \rho^{\prime}\left(m \cdot e^{\prime}\right)}|\rho|^{d_{v}-2}\left|\rho^{\prime}\right|^{d_{v}-2} d \rho d \rho^{\prime}
\end{aligned}
$$

in polar coordinates. We make the decoupling assumption that

$$
\tilde{B}(|x|,|y|)=a(|x|) b(|y|),
$$

then

$$
\begin{aligned}
& \beta(l, m)=\frac{1}{4} \int_{e, e^{\prime} \in \mathbb{S} d_{v}-1, e \perp e^{\prime}} d e d e^{\prime} \\
& \cdot\left(\int_{-R}^{R} a(|\rho|)|\rho|^{d_{v}-2} e^{i \frac{\pi}{L} \rho(l \cdot e)} d \rho\right)\left(\int_{-R}^{R} b\left(\left|\rho^{\prime}\right|\right)\left|\rho^{\prime}\right|^{d_{v}-2} e^{i \frac{\pi}{L} \rho^{\prime}\left(m \cdot e^{\prime}\right)} d \rho^{\prime}\right) \\
& =\frac{1}{4} \int_{e, e^{\prime} \in \mathbb{S}^{d}-1, e \perp e^{\prime}} \phi(l \cdot e) \phi^{\prime}\left(m \cdot e^{\prime}\right) d e d e^{\prime},
\end{aligned}
$$

where

$$
\begin{aligned}
\phi(s) & \doteq \int_{-R}^{R} a(|\rho|)|\rho|^{d_{v}-2} e^{i \frac{\pi}{L} \rho s} d \rho, \\
\phi^{\prime}(s) & \doteq \int_{-R}^{R} b(|\rho|)|\rho|^{d_{v}-2} e^{i \frac{\pi}{L} \rho s} d \rho .
\end{aligned}
$$


- In the 2-D case,

$$
\beta(l, m)=\int_{0}^{\pi} \phi\left(l \cdot e_{\theta}\right) \phi^{\prime}\left(m \cdot e_{\theta+\frac{\pi}{2}}\right) d \theta, \quad e_{\theta}=(\cos \theta, \sin \theta),
$$

so we can approximate it by

$$
\beta(l, m) \approx \frac{\pi}{M} \sum_{p=1}^{M} \phi\left(l \cdot e_{\theta_{p}}\right) \phi^{\prime}\left(m \cdot e_{\theta_{p}+\frac{\pi}{2}}\right),
$$

where $M$ is the number of equally spaced points in $[0, \pi]$.

- In the 3-D case,

$$
\beta(l, m)=\int_{e \in \mathbb{S}_{+}^{2}} \phi(l \cdot e) \psi\left(\left|\Pi_{e^{\perp}}(m)\right|\right) d e, \quad \psi(s) \doteq \int_{0}^{\pi} \phi^{\prime}(s \cos \eta) \sin \eta d \eta
$$

where $\Pi_{e^{\perp}}$ is the orthogonal projection onto the plane $e^{\perp}$.

Parametrizing $e$ as $e=(\sin \varphi \cos \theta, \sin \varphi \sin \theta, \cos \varphi), \theta, \varphi \in[0, \pi]$, we can approximate $\beta$ by

$$
\beta(l, m) \approx\left(\frac{\pi}{M}\right)^{2} \sum_{p, q=1}^{M} \phi\left(l \cdot e_{\theta_{p}, \varphi_{q}}\right) \psi\left(\left|\Pi_{e_{\theta_{p}, \varphi_{q}}^{\perp}}(m)\right|\right) \sin \varphi_{q},
$$

$M$ is the same as in 2-D.

2.2. A fast algorithm. Based on previous discussions we see that the decomposition (2.13) is good for the fast evaluation of $\mathcal{Q}_{c}$ (see [11]), $\mathcal{Q}_{2}, \mathcal{Q}_{3}$, and $\mathcal{Q}_{4}$. To improve the computation of $\mathcal{Q}_{1}$, we need to seek other decompositions.

Suppose we can write $\beta$ as follows:

$$
\beta(l, m) \approx \sum_{j=1}^{J} \exp \left(i \alpha_{j}(l)\right) \alpha_{j}^{\prime}(m),
$$

where $\alpha_{j}$ is assumed to be linear in $l$. Then one can compute $\mathcal{Q}_{1}$ efficiently by using the fundamental property of the exponential function: $e^{a+b}=e^{a} e^{b}$. In fact, (2.14) becomes

$$
\begin{aligned}
\hat{\mathcal{Q}}_{1, k} & =\sum_{\substack{l, m, n=-\frac{N}{2} \\
l+m+n=k}}^{\frac{N}{2}-1} \beta(l+n, m+n) \hat{f}_{l} \hat{f}_{m} \hat{f}_{n} \\
& \approx \sum_{\substack{j=1 \\
l, m, n=-\frac{N}{2} \\
l+m+n=k}}^{J} \sum_{\substack{\frac{N}{2}-1 \\
l}} \exp \left(i \alpha_{j}(l)\right) \exp \left(i \alpha_{j}(n)\right) \alpha_{j}^{\prime}(m+n) \hat{f}_{l} \hat{f}_{n} \hat{f}_{m} \\
& =\sum_{j=1}^{J} \sum_{l=-\frac{N}{2}}^{\frac{N}{2}-1} \exp \left(i \alpha_{j}(l)\right) \alpha_{j}^{\prime}(k-l)\left[\sum_{\substack{2 \\
m, n=-\frac{N}{2} \\
m+n=k-l}}^{\frac{N}{2}-1} \exp \left(i \alpha_{j}(n)\right) \hat{f}_{n} \hat{f}_{m}\right] \hat{f}_{l},
\end{aligned}
$$

so it is again in the double-convolution form and can be evaluated by the FFT. 
Fortunately, the decomposition (2.27) can be readily obtained thanks to the special form of $\phi(s)$ (2.21). Specifically, we still adopt the discretizations (2.24) and (2.26), but instead of precomputing $\phi(s)$, we approximate it by a numerical quadrature rule:

$$
\phi(s) \approx \sum_{j=1}^{J}\left[a\left(\left|\rho_{j}\right|\right)\left|\rho_{j}\right|^{d_{v}-2} \exp \left(i \frac{\pi}{L} \rho_{j} s\right)\right] \cdot w_{j},
$$

where $\rho_{j}, w_{j}, j=1, \ldots J$ are the integration points and weights in $[-R, R]$. Therefore,

- in the 2-D case,

$$
\beta(l, m) \approx \frac{\pi}{M} \sum_{p=1}^{M} \sum_{j=1}^{J}\left[a\left(\left|\rho_{j}\right|\right) \exp \left(i \frac{\pi}{L} \rho_{j}\left(l \cdot e_{\theta_{p}}\right)\right) \phi^{\prime}\left(m \cdot e_{\theta_{p}+\frac{\pi}{2}}\right)\right] \cdot w_{j} ;
$$

- in the 3-D case,

$$
\begin{array}{r}
\beta(l, m) \approx\left(\frac{\pi}{M}\right)^{2} \sum_{p, q=1}^{M} \sum_{j=1}^{J}\left[a\left(\left|\rho_{j}\right|\right)\left|\rho_{j}\right| \exp \left(i \frac{\pi}{L} \rho_{j}\left(l \cdot e_{\theta_{p}, \varphi_{q}}\right)\right)\right. \\
\left.\cdot \psi\left(\left|\Pi_{e_{\theta_{p}, \varphi_{q}}}(m)\right|\right) \sin \varphi_{q}\right] \cdot w_{j} .
\end{array}
$$

In our implementation, we use the Gauss quadrature or composite Gauss quadrature for the integration depending on the properties of $a(|\rho|)|\rho|^{d_{v}-2}$. For reasonably nice $a(|\rho|)|\rho|^{d_{v}-2}$, the integrand of $\phi(l \cdot e)$ oscillates on the scale of $O(N)$ for $l \in\left[-\frac{N}{2}, \frac{N}{2}-1\right]$, so $J$ should be taken as $O(N)$. Thus the total number of terms in the decomposition of $\beta$ is equal to $M^{d_{v}-1} J=O\left(M^{d_{v}-1} N\right)$.

We are ready to summarize the final algorithm for computing $\mathcal{Q}_{q}(f)$. Given $f(v)$ at a fixed time $t$,

1. Compute $\hat{f}_{k}$ by the FFT.

2. Evaluate $\mathcal{Q}_{c}, \mathcal{Q}_{2}, \mathcal{Q}_{3}$, and $\mathcal{Q}_{4}$ by the method presented in Section 2.1, with $\beta(l, m)$ given by the formulas (2.24) and (2.26).

3. Evaluate $\mathcal{Q}_{1}$ by the method presented in this subsection, with $\beta(l, m)$ given by the formulas (2.30) and (2.31).

4. Construct $\mathcal{Q}_{q}$ through (2.2).

The main work load still comes from step 3, which is now reduced to $O\left(M^{d_{v}-1} N^{d_{v}+1} \log N\right)$.

\section{Numerical tests}

In this section we provide some numerical results of our new algorithm. We first test its performance on the steady state, i.e. compute $\mathcal{Q}_{q}\left(\mathcal{M}_{q}\right)$ and check the accuracy. Fixing the macroscopic quantities: density $\rho=1$, temperature $T=1$, macrovelocity $u=0$, one can adjust $\theta_{0}$ to get $\mathcal{M}_{q}$ that lies in different physical regimes [9]. We consider the 2-D Maxwellian molecules and 3-D hard sphere models which both satisfy the decoupling assumption (2.19), as $\tilde{B}$ is a constant in either case (without loss of generality, assume $a=b=1)$. The computational domain for $v$ is taken as $[-8,8]^{d_{v}}$. The Gauss-Legendre quadrature rule with $J=O(N)$ is employed to integrate $\phi(s)$. In the 3 -D case, due to the kink $|\rho|$ in the integrand we divide $[-R, R]$ to $[-R, 0]$ and $[0, R]$, and apply the Gauss quadrature on each interval.

The implementation is in MATLAB and all the numerical results are obtained on a desktop computer with $2.8 \mathrm{GHz}$ CPU. Further acceleration can be achieved by careful implementation in $\mathrm{C}$ or Fortran. 
3.1. 2-D Maxwellian molecules. In this case

$$
\phi(s)=\phi^{\prime}(s)=2 R \operatorname{Sinc}\left(\frac{\pi}{L} R s\right)
$$

where $\operatorname{Sinc}(x)=\sin x / x$.

Let $\theta_{0}=0.1^{2} \quad($ Planck constant $h=0.1)$, then $z_{\text {Bose }}=1.5903 e-03, \quad z_{\text {Fermi }}=$ $1.5928 e-03$. Here $z \ll 1$ implies that the quantum effect is very small. Either the Bose gas or Fermi gas should behave like a classical gas. When we increase $\theta_{0}$, say $\theta_{0}=3^{2}(h=3)$, then $z_{\text {Bose }}=0.7613, z_{\text {Fermi }}=3.1887$. Now the difference between the quantum gases and classical gas is evident (Figure 3.1).

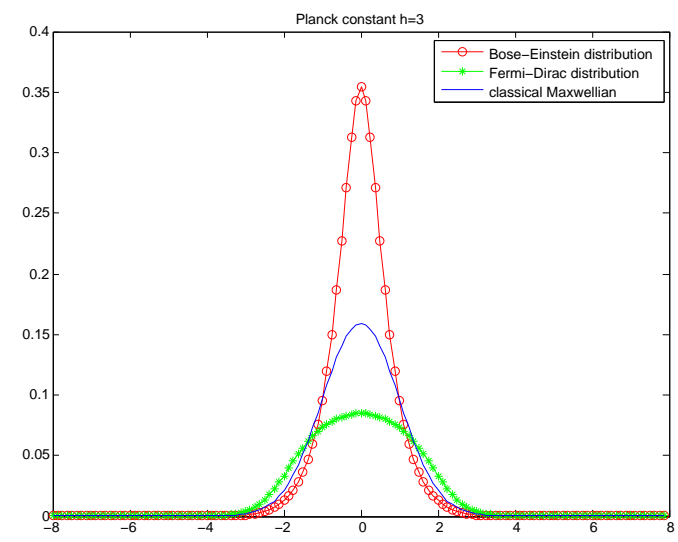

FiG. 3.1. The cross section of the Maxwellians when $\theta_{0}=3^{2}$.

Tables 3.1 and 3.2 report the max norms of $\mathcal{Q}_{q}\left(\mathcal{M}_{q}\right)$ generated on different meshes. For comparison we also list the values computed by the spectral method without the speedup strategy for $\mathcal{Q}_{1}$ (we call it direct method). Clearly our new algorithm gives accuracy comparable to the direct method. Table 3.3 shows the average running time of both methods for computing $\mathcal{Q}_{1}$. The speedup factor is more than 100 in $N=128$ case.

\begin{tabular}{c|c|c|c|c}
\hline$N$ & fast, Bose $(J=N)$ & direct, Bose & fast, Fermi $(J=N)$ & direct, Fermi \\
\hline 16 & $1.2301 \mathrm{e}-04$ & $1.1201 \mathrm{e}-04$ & $1.1973 \mathrm{e}-04$ & $1.3060 \mathrm{e}-04$ \\
32 & $5.3865 \mathrm{e}-10$ & $4.1312 \mathrm{e}-10$ & $4.7734 \mathrm{e}-10$ & $3.6743 \mathrm{e}-10$ \\
64 & $4.8451 \mathrm{e}-12$ & $4.8451 \mathrm{e}-12$ & $4.8606 \mathrm{e}-12$ & $4.8606 \mathrm{e}-12$ \\
128 & $4.8643 \mathrm{e}-12$ & $4.8643 \mathrm{e}-12$ & $4.8798 \mathrm{e}-12$ & $4.8798 \mathrm{e}-12$ \\
\hline
\end{tabular}

TABLE 3.1. $\left\|\mathcal{Q}_{q}\left(\mathcal{M}_{q}\right)\right\|_{L} \infty$ in the classical regime $\left(\theta_{0}=0.1^{2}\right)$ computed by the fast algorithm and the direct method. $M=8$ in all the simulations.

3.2. 3-D hard sphere models. In this case

$$
\phi(s)=R^{2}\left[2 \operatorname{Sinc}\left(\frac{\pi}{L} R s\right)-\operatorname{Sinc}^{2}\left(\frac{\pi}{2 L} R s\right)\right], \quad \psi(s)=2 R^{2} \operatorname{Sinc}\left(\frac{\pi}{2 L} R s\right) .
$$

We test the fast algorithm in (1) the classical regime: $\theta_{0}=0.3^{3}$ (Planck constant $h=0.3$ ), which corresponds to $z_{\text {Bose }}=1.7133 e-03, z_{\text {Fermi }}=1.7154 e-03$; and $(2)$ the 


\begin{tabular}{c|c|c|c|c}
\hline$N$ & fast, Bose $(J=N)$ & direct, Bose & fast, Fermi $(J=N)$ & direct, Fermi \\
\hline 16 & 0.0767 & 0.4576 & $3.9164 \mathrm{e}-04$ & 0.0014 \\
32 & 0.0034 & 0.0248 & $1.3642 \mathrm{e}-06$ & $2.8704 \mathrm{e}-06$ \\
64 & $1.0880 \mathrm{e}-05$ & $1.2762 \mathrm{e}-04$ & $5.3347 \mathrm{e}-11$ & $1.0608 \mathrm{e}-10$ \\
128 & $4.5895 \mathrm{e}-10$ & $4.6890 \mathrm{e}-09$ & $2.4142 \mathrm{e}-11$ & $2.4142 \mathrm{e}-11$ \\
\hline
\end{tabular}

TABLE 3.2. $\left\|\mathcal{Q}_{q}\left(\mathcal{M}_{q}\right)\right\|_{L^{\infty}}$ in the quantum regime $\left(\theta_{0}=3^{2}\right)$ computed by the fast algorithm and the direct method. $M=8$ in all the simulations.

\begin{tabular}{c|c|c}
\hline$N$ & fast algorithm $(J=N)$ & direct method \\
\hline 16 & $0.04 \mathrm{~s}$ & $0.36 \mathrm{~s}$ \\
32 & $0.09 \mathrm{~s}$ & $2.30 \mathrm{~s}$ \\
64 & $0.55 \mathrm{~s}$ & $28.91 \mathrm{~s}$ \\
128 & $2.43 \mathrm{~s}$ & $341.45 \mathrm{~s}$ \\
\hline
\end{tabular}

TABLE 3.3. The average running time of the fast algorithm and the direct method for computing $\mathcal{Q}_{1} . M=8$ in all the simulations.

\begin{tabular}{c|c|c|c|c}
\hline$N$ & fast, Bose $(J=N)$ & direct, Bose & fast, Fermi $(J=N)$ & direct, Fermi \\
\hline 8 & 0.0886 & 0.0884 & 0.0884 & 0.0886 \\
16 & $1.4853 \mathrm{e}-04$ & $1.4245 \mathrm{e}-04$ & $1.4225 \mathrm{e}-04$ & $1.4825 \mathrm{e}-04$ \\
32 & $6.3897 \mathrm{e}-10$ & $3.4993 \mathrm{e}-09$ & $5.6455 \mathrm{e}-10$ & $3.3125 \mathrm{e}-09$ \\
64 & $4.2374 \mathrm{e}-12$ & - & $4.2477 \mathrm{e}-12$ & - \\
128 & $4.2374 \mathrm{e}-12$ & - & $4.2477 \mathrm{e}-12$ & - \\
\hline
\end{tabular}

TABLE 3.4. $\left\|\mathcal{Q}_{q}\left(\mathcal{M}_{q}\right)\right\|_{L^{\infty}}$ in the classical regime $\left(\theta_{0}=0.3^{3}\right)$ computed by the fast algorithm and the direct method. $M=8$ in all the simulations.

\begin{tabular}{c|c|c|c|c}
\hline$N$ & fast, Bose $(J=N)$ & direct, Bose & fast, Fermi $(J=N)$ & direct, Fermi \\
\hline 8 & 1.8267 & 3.8975 & 0.0106 & 0.0305 \\
16 & 0.0214 & 0.1752 & $6.3556 \mathrm{e}-04$ & 0.0013 \\
32 & $9.1508 \mathrm{e}-04$ & 0.0036 & $9.7828 \mathrm{e}-07$ & $1.1671 \mathrm{e}-06$ \\
64 & $2.2308 \mathrm{e}-06$ & - & $3.5756 \mathrm{e}-11$ & - \\
128 & $4.2658 \mathrm{e}-11$ & - & $1.3868 \mathrm{e}-11$ & - \\
\hline
\end{tabular}

TABLE 3.5. $\left\|\mathcal{Q}_{q}\left(\mathcal{M}_{q}\right)\right\|_{L^{\infty}}$ in the quantum regime $\left(\theta_{0}=2.6^{3}\right.$ for Bose gas; $\theta_{0}=3^{3}$ for Fermi gas) computed by the fast algorithm and the direct method. $M=8$ in all the simulations.

quantum regime: $\theta_{0}=2.6^{3}(h=2.6)$ for the Bose gas, $z_{\text {Bose }}=0.7474 ; \theta_{0}=3^{3} \quad(h=3)$ for the Fermi gas, $z_{\text {Fermi }}=3.0997$. The numerical results of both methods are given in Tables 3.4-3.6. In the 3-D case, the fast algorithm provides a huge saving of the computational time, while the direct method is too slow.

3.3. Relaxation to equilibrium. In this subsection, we would like to test the numerical performance of the algorithm on a general distribution $f$, instead of $\mathcal{M}_{q}$. Consider the homogeneous Equation (2.1) for a 2-D Bose gas of Maxwellian 


\begin{tabular}{c|c|c}
\hline$N$ & fast algorithm $(J=N)$ & direct method \\
\hline 8 & $0.08 \mathrm{~s}$ & $5.04 \mathrm{~s}$ \\
16 & $0.99 \mathrm{~s}$ & $236.23 \mathrm{~s}$ \\
32 & $9.49 \mathrm{~s}$ & $\sim 12000 \mathrm{~s}$ \\
64 & $144.31 \mathrm{~s}$ & - \\
128 & $\sim 1900 \mathrm{~s}$ & - \\
\hline
\end{tabular}

TABLE 3.6. The average running time of the fast algorithm and the direct method for computing $\mathcal{Q}_{1} . M=8$ in all the simulations.
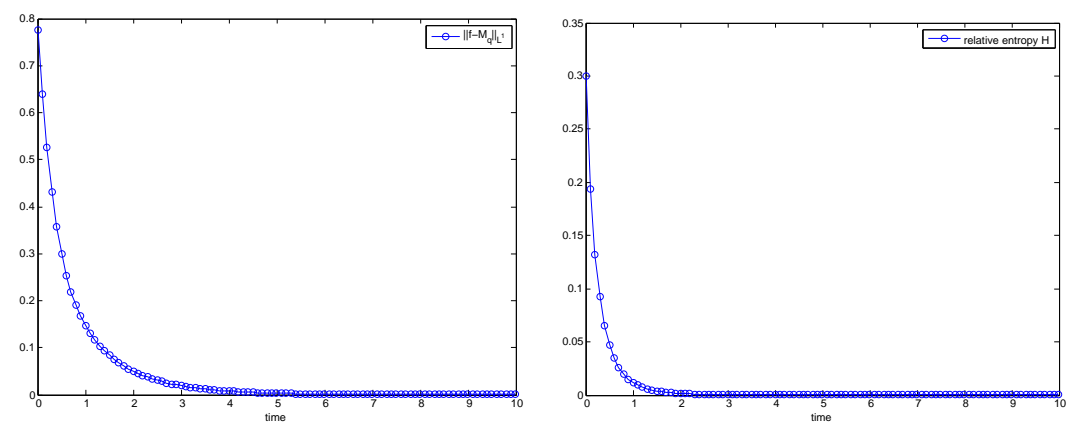

FIG. 3.2. The time evolution of $\left\|f-\mathcal{M}_{q}\right\|_{L^{1}}$ and the relative entropy $H$ for a 2-D Bose gas.
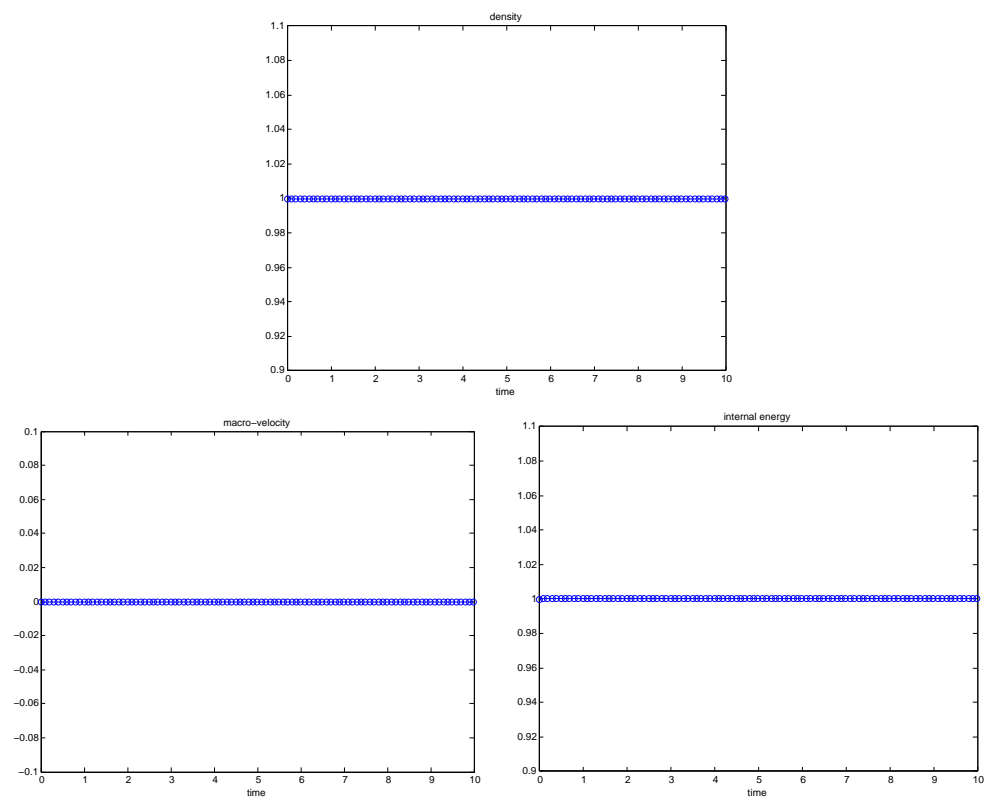

FIG. 3.3. The time evolution of density, macro-velocity, and specific internal energy for a 2-D Bose gas. 
molecules, with the nonequilibrium initial data

$$
f_{0}(v)=\frac{\rho_{0}}{4 \pi T_{0}}\left(\exp \left(-\frac{\left|v-u_{0}\right|^{2}}{2 T_{0}}\right)+\exp \left(-\frac{\left|v+u_{0}\right|^{2}}{2 T_{0}}\right)\right),
$$

where $\rho_{0}=1, T_{0}=3 / 8$, and $u_{0}=(1,1 / 2)$. The fast algorithm for $\mathcal{Q}_{q}$ and the fourthorder Runge-Kutta method for the time derivative are applied to solve the equation. Here the Planck constant $h$ is chosen as 3 such that $z=0.6635$. The computational domain is $[-8,8]^{2}$. Here $N=64=J$ and $M=8$. Figure 3.2 shows the time evolution of $\left\|f-\mathcal{M}_{q}\right\|_{L^{1}}$ and the relative entropy $H$, from which we clearly observe the relaxation to equilibrium and the entropy decay. Furthermore, the mass, momentum, and energy are also preserved very well (Figure 3.3).

\section{Conclusion}

We improved the spectral method in [5] and developed a fast algorithm for the quantum Boltzmann collision operator. The special form of the collision kernel in the spatial domain enables us to accelerate the computation. With the fast Fourier transform, the algorithm complexity is brought down to $O\left(M^{d_{v}-1} N^{d_{v}+1} \log N\right)$ as opposed to $O\left(M^{d_{v}-1} N^{2 d_{v}} \log N\right)$ of the previous method. Numerical results showed that the new algorithm preserves the spectral accuracy as well as offers a significant speedup over the original approach.

\section{REFERENCES}

[1] G.A. Bird, Molecular Gas Dynamics and the Direct Simulation of Gas Flows, Clarendon Press, Oxford, 1994.

[2] A.V. Bobylev, A. Palczewski, and J. Schneider, On approximation of the Boltzmann equation by discrete velocity models, C.R. Acad. Sci. Paris Sér. I Math., 320, 639-644, 1995.

[3] A.V. Bobylev and S. Rjasanow, Fast deterministic method of solving the Boltzmann equation for hard spheres, European J. Mech. B Fluids, 18, 869-887, 1999.

[4] C. Buet, A discrete velocity scheme for the Boltzmann operator of rarefied gas dynamics, Transp. Theory Stat. Phys., 25, 33-60, 1996.

[5] F. Filbet, J.W. Hu, and S. Jin, A numerical scheme for the quantum Boltzmann equation with stiff collision terms, ESAIM-Math. Model. Numer. Anal., 46, 443-463, 2012.

[6] F. Filbet, C. Mouhot, and L. Pareschi, Solving the Boltzmann equation in $N \log N$, SIAM J. Sci. Comput., 28, 1029-1053, 2006.

[7] I.M. Gamba and S.H. Tharkabhushanam, Spectral-Lagrangian methods for collisional models of non-equilibrium statistical states, J. Comput. Phys., 228, 2012-2036, 2009.

[8] A.L. Garcia and W. Wagner, Direct simulation Monte Carlo method for the Uehling-UhlenbeckBoltzmann equation, Phys. Rev. E, 68, 056703, 2003.

[9] J.W. Hu and S. Jin, On kinetic flux vector splitting schemes for quantum Euler equations, Kinet. Relat. Models, 4, 517-530, 2011.

[10] P. Markowich and L. Pareschi, Fast, conservative and entropic numerical methods for the Bosonic Boltzmann equation, Numerische Math., 99, 509-532, 2005.

[11] C. Mouhot and L. Pareschi, Fast algorithms for computing the Boltzmann collision operator, Math. Comput., 75, 1833-1852, 2006.

[12] K. Nanbu, Direct simulation scheme derived from the Boltzmann equation. I. Monocomponent gases, J. Phys. Soc. Japan, 52, 2042-2049, 1983.

[13] L.W. Nordheim, On the kinetic method in the new statistics and its application in the electron theory of conductivity, Proc. R. Soc. London, Ser. A, 119, 689-698, 1928.

[14] L. Pareschi and B. Perthame, A Fourier spectral method for homogeneous Boltzmann equations, Transp. Theory Stat. Phys., 25, 369-383, 1996.

[15] L. Pareschi and G. Russo, Numerical solution of the Boltzmann equation I. Spectrally accurate approximation of the collision operator, SIAM J. Numer. Anal., 37, 1217-1245, 2000.

[16] F. Rogier and J. Schneider, A direct method for solving the Boltzmann equation, Transp. Theory Stat. Phys., 23, 313-338, 1994.

[17] E.A. Uehling and G.E. Uhlenbeck, Transport phenomena in Einstein-Bose and Fermi-Dirac gases I, Phys. Rev., 43, 552-561, 1933. 